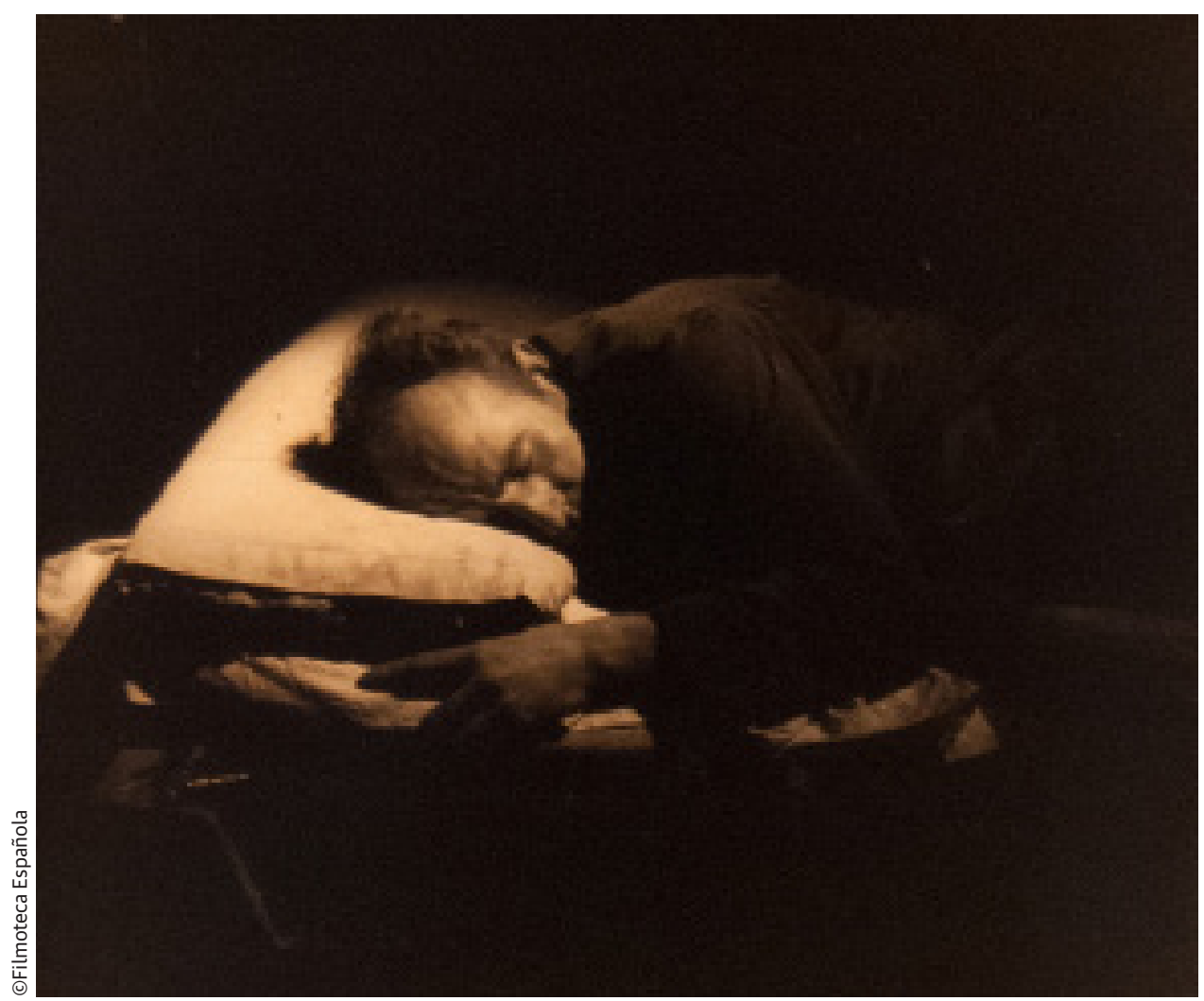

\title{
EL INSTITUTO ANTES DE SALAMANCA. LOS PRIMEROS AÑOS DEL INSTITUTO DE INVESTIGACIONES Y EXPERIENCIAS CINEMATOGRÁFICAS (1947-1955)
}

THE INSTITUTO BEFORE SALAMANCA. THE INSTITUTO DE INVESTIGACIONES Y EXPERIENCIAS CINEMATOGRÁFICAS’ FIRST YEARS (1947-1955)

Fernando Ramos Arenas / fernando.ramos@uni-leipzig.de UNIVERSIDAD DE LEIPZIG 


\section{RESUMEN}

El presente texto analiza la función del Instituto de Investigaciones y Experiencias Cinematográficas (IIEC) como punto de encuentro de la cultura cinematográfica nacional entre 1947 y 1955 -los años de Victoriano López- y dirige el foco analítico a los discursos, debates e intereses que marcaron la labor del instituto en estos primeros años. Para ello, este artículo fija su atención en uno de los pocos documentos originales conservados de esa época, un boletín publicado por los alumnos en 1951, material que será aquí completado con entrevistas y los escasos documentos referentes al instituto conservados en el Archivo General de la Administración. La piedra de toque del análisis la constituyen por un lado las actividades del cineclub, organizado por los alumnos de la clase de Historia del Cine en 1951, y, en el plano discursivo, los debates en torno a la Filmología, doctrina con la que desde 1947 se intenta dotar a las actividades del Instituto de una base teórica con desiguales resultados. Se consigue así un acercamiento a las prácticas y discursos esenciales para comprender la importancia del instituto como punto central dentro de una naciente cultura cinematográfica nacional.

PALABRAS CLAVE

IIEC, cineclub, cultura cinematográfica, cinefilia, Filmología

\section{ABSTRACT}

This article analyses the role of the Instituto de Investigaciones y Experiencias Cinematográficas (IIEC) as a meeting point for Spanish national filmmaking between 1947 and 1955 -Victoriano López's years- and focuses on the discourses, debates and interests that characterized the Instituto's early years. It therefore directs its attention towards one of the few original sources that have been preserved, a bulletin published by the Instituto's students in 1951. This documentation has been supplemented with interviews and the few documents about the Instituto kept in the government's general archive. The analysis' cornerstones are, on the one hand, the film club's activities, organized by students in the 1951 Film History, and, on a theoretical level, debates around the subject of Filmology, a theory used from 1947 as an attempt to supply the Instituto with a theoretical backbone, with uneven results. Through this process we can approach with first hand information these experiences and essential discourses to better understand the importance of the Instituto as the epicenter of an emerging Spanish filmmaking culture.

KEYWORDS

IIEC, Film Club, Film Culture, Cinephile, Filmology

Recibido: 28 de marzo de 2016

Aceptado: 15 de junio de 201 


\section{INTRODUCCIÓN}

Entre 1947, año de su fundación, y 1955, cuando su iniciador Victoriano López García es sustituido por José Cano Lechuga, el Instituto de Investigaciones y Experiencias Cinematográficas (IIEC) constituyó, más allá de su labor como centro de enseñanza, la clave de bóveda de una paupérrima cultura cinematográfica nacional. Estos ocho primeros años de vida son un periodo esencial para comprender el posterior desarrollo de la institución: en gran medida marginada por las autoridades estatales e ignorada por una industria donde los estudiantes difícilmente conseguían trabajo. El instituto se constituiría en esta primera época en un lugar de encuentro de jóvenes cinéfilos; un punto de convergencia de diferentes tradiciones y generaciones dentro de una cultura cinematográfica apenas recuperada del parón bélico: los cineclubs -salvo las contadas excepciones como el de Zaragoza, algunos en la órbita del SEU o el del Círculo de Escritores Cinematográficos- eran en gran medida una rara avis, la Filmoteca no será fundada hasta 1953 y las revistas especializadas más significativas se hacen igualmente esperar: Objetivo aparece en 1953, Cinema Universitario en 1955 y Film Ideal en 1956.

Con anterioridad a esta renovación de mediados de los años cincuenta que habría de concretarse simbólicamente en la celebración de las Conversaciones de Salamanca, el instituto se encuentra entre dos planos: una generación que había descubierto el cine en los años anteriores a la Guerra Civil y un grupo de jóvenes que empieza a preparar la ruptura de los años venideros, una modernidad que internacionalmente había surgido con el Neorrealismo, pero que en España era aún desconocida.

El cese de Victoriano López en 1955 ocurre en un momento que acentúa su significación simbólica en el ámbito cultural franquista. Dentro del propio instituto, culmina un movimiento de protesta con numerosas huelgas en el curso 1954-55, sobre todo por problemas de falta de medios, de material e instalaciones y un descontento general con la actuación de la Dirección
General de Cinematografía y Teatro (DGCT) ${ }^{1}$. No obstante, los conflictos en el IIEC ganan en relevancia como prolegómeno a las protestas universitarias de 1956 y de un gradual cuestionamiento ideológico del franquismo en el ámbito educativo (Rodríguez Tejada, 2015: 85f).

El presente texto no pretende pues escribir una historia institucional al uso como listas de alumnos y profesores, planes de estudio o legislación; tampoco analizar sus producciones o recapitular los recuerdos de los asistentes. Trata más bien de resaltar su función como punto de encuentro de la cultura cinematográfica nacional y dirigir así el foco analítico a los discursos, debates e intereses que marcaron la labor del centro en estos primeros años. El principal problema al investigar este periodo es la falta de fuentes contemporáneas y de primera mano. Numerosos son los testimonios de algunos de los protagonistas -estudiantes y profesoresque pasaron por las aulas 2 pero casi inexistente el material producido desde el instituto que nos permita comprender mejor su funcionamiento. Este artículo fija su atención en uno de estos pocos documentos originales conservados de esa época, un boletín publicado por los alumnos en $1951^{3}$, material que será aquí completado con entrevistas y los escasos textos conservados en el Archivo General de la Administración. La piedra de toque del análisis la constituyen por un lado las actividades del cineclub, organizado por los alumnos de la clase de Historia del Cine en 1951 $\mathrm{y}$, en el plano teórico, los debates en torno a la

\footnotetext{
1 De valor simbólico es en este sentido, junto a los numerosos testimonios recogidos en el anexo de la tesis doctoral de Blanco Mallada IIEC y EOC: Una escuela para el cine español (1990), la "Solicitud efectuada por la Escuela Especial de Ingenieros Industriales para que se le conceda subvención para mejorar su laboratorio de cine", conservada en el Archivo del Ministerio de Cultura (Caja AGA 12); formulada al final del periodo aquí analizado (7 de febrero de 1956) y que fue denegada.

2 Muchos de ellos recogidos en el estudio de Lucio Blanco Mallada. Esta tesis doctoral no publicada es imprescindible para conocer algunos datos esenciales referentes a la historia del instituto y la escuela. Otros testimonios de valor se encuentran también en el volumen 50 años de la escuela de cine, publicado por la Filmoteca Española.
}

3 En el archivo de la Filmoteca Española: AMO/01/118. 
Filmología, doctrina con la que desde 1947 se intenta dotar a las actividades del instituto de una base teórica con desiguales resultados.

Este texto plantea la interpretación de este material dentro del (re)nacimiento de una tradición cinéfila española que a lo largo de la década de los cincuenta habría de ganar en relevancia, diferenciación y autonomía dando lugar a un campo cultural caracterizado por la emergencia de: a) instituciones tales como una extensa red de cineclubs o publicaciones, festivales, etc., asî como b) discursos especializados que remitían a tendencias internacionales - politique des auteurs, debates sobre el realismo, teorización y politización de la crítica cinematográfica-, a su vez adaptados y transformados de acuerdo con la realidades culturales bajo la dictadura. Instituciones y discursos que, junto con individuos concretos, prepararían y acompañarían la llegada de una modernidad fílmica a lo largo de los siguientes veinte años ${ }^{4}$. Así, al bosquejar la necesidad de repensar la Historia del Cine también como una Historia Cultural, este texto se enmarca dentro de las corrientes metodológicas más actuales en el campo de los Film Studies (New Film History), al mismo tiempo que las amplía y fija su atención en un caso que, por las condiciones políticas y culturales del país en cuestión, plantea interesantes consideraciones acerca del papel social del cine, entendido esencialmente como artefacto social, pantalla de proyección para discursos, tensiones y conflictos que en muchas ocasiones van más allá de las películas en sí.

\section{UN PUNTO DE ENCUENTRO PARA UNA GENERACIÓN}

Cuando en 1947 aparece el centro se está dando naturaleza institucional a una serie muy diversa de líneas de tradición dentro de la cultura cinematográfica española de los años cuarenta. En él toma forma el creciente interés en los aspectos técnicos del cine que se había visto reflejado en unos cursos impartidos en la Escuela de Ingenieros Industriales desde $1942^{5}$. A estos se unen también las lecciones esporádicas que desde 1945 Joaquín de Entrambasaguas impartía en la Facultad de Filosofía y Letras. Victoriano López - profesor en la Escuela de Ingenieros Industriales- encontrará muchos interesados en ayudarle en su proyecto de escuela: en la industria (Luis Marquina, José María Elorrieta), en la Universidad (José Camón Aznar, profesor de Historia del Arte en la Facultad de Filosofĩa y Letras), en la crítica cinematográfica (Luis Gómez Mesa) y en los círculos en torno a Cine Experimental. Esta publicación minoritaria y de corta duración -solo doce números, 1946-48, pero de capital importancia en ambientes cinematográficos- informó de la construcción de un Laboratorio de Investigaciones Cinematográficas en la Escuela de Ingenieros e impulsó desde sus páginas la necesidad de crear un centro para la formación de nuevos técnicos. Además de estas personas se sumaría Carlos Serrano de Osma que llegaría a ser profesor del mismo.

Las clases se celebran en estos primeros años en las aulas de Escuela Superior de Ingenieros Industriales en los Altos del Hipódromo, en lo que era entonces el final de la Castellana. El instituto está pobremente equipado para la práctica y parte del trabajo en laboratorios y estudios tiene lugar, por ejemplo, en instalaciones exteriores. A estas deficiencias se unen problemas de orden administrativo que dificultarán las relaciones entre el instituto y la industria: unas Órdenes Ministeriales habían creado y regulado los primeros pasos el 18 y el 26 de febrero de 1947; el Patronato de Divulgaciones y Experiencias Cinematográficas aprobará un reglamento en $1949^{6}$ y solamente dos años después, el 18 de mayo de

\footnotetext{
4 Véase en este sentido la reciente publicación Crónica de un desencuentro. La recepción del cine moderno en España (Monterde y Piñol, 2015)

5 En 1942 la Escuela Especial de Ingenieros Industriales incluye en su plan de estudios la asignatura de Cinematografía; en 1943 se crea un Laboratorio de Cinematografía, con estudio, laboratorio de sonido, revelado, montaje, sensitometría y óptica, base del instituto a partir de 1947.

6 Número 2 del boletín. Al no estar sus páginas numeradas, las citas del boletín harán referencia de aquí en adelante simplemente al número.
} 
1951, se crea un diploma de Cinematografía para aquellos alumnos de las diferentes especialidades que están a punto de terminar sus estudios. Este diploma tendrá desde entonces validez para el Ministerio de Educación Nacional pero no será reconocido por el Sindicato Nacional del Espectáculo, lo que implica que el camino profesional está cerrado para los egresados.

$\mathrm{El}$ incierto futuro profesional es el "superproblema" de los estudiantes de acuerdo con un texto de Eduardo Ducay (El Instituto y nosotros), que apareció en el segundo número del boletín. Lo resume con las siguientes palabras: "de nosotros, ¿quién se va a necesitar? El libre ejercicio de nuestra futura profesión es una cosa que encontramos muy difícil en este mundo del cine español en que tan poca atención se nos concede". No se trata una queja puntual: año y medio más tarde Jesús Franco publica en Índice (diciembre 1952) un artículo, Huelga en el Instituto, sobre las dificultades de los estudiantes de la IIEC para encontrar ayuda (Salvador Marañón, 2006: 92). Y todo esto pese a que es entonces cuando las primeras generaciones abandonan el instituto y algunos de sus representantes tendrán una cierta actividad en torno a la productora Altamira. No obstante, el "superproblema" solo empezará a solucionarse con la llegada a la dirección de José Luis Sáenz de Heredia en marzo de 1959. Aprovechará su posición en la industria para aumentar la visibilidad del centro, organizará proyecciones públicas y llevará las películas de los alumnos a las Jornadas Internacionales de las Escuelas de Cinematografía que se celebran anualmente a partir de 1960 en torno al Festival Internacional de Cine de San Sebastián. En esta primera edición comparecen representando al Instituto José Luis Borau, Basilio Martín Patino, Miguel Picazo, Manuel Summers y Francisco Prósper (Heredero, 1993: 307).

La financiación insuficiente de las prácticas, la falta de reconocimiento del diploma o la misma creación de este en fecha tan tardía como 1951 son, además, hechos significativos en cuanto que muestran una tendencia más general de la administración con respecto a la IIEC: una gran falta de interés por sus actividades. Este desinterés permitió la aparición de un espacio de desencuentro con el régimen. No se pretende afirmar que existiera un intento consciente desde el franquismo de aceptar una isla ideológica liberal en los primeros años, de una izquierda posibilista en torno a 1960 o radical a partir de mediados de la década de los sesenta y setenta. Pero lo que sí se crea, de acuerdo con la mayoría de los testimonios, es un espacio en el que el dogmatismo político está fuera de lugar; se ponen así las líneas maestras de un estatus quo asumido por alumnos y profesores, un lugar de encuentro pero también de colisión entre distintas formas de entender la labor del centro.

De hecho, no son pocos, estudiantes y profesores, los que se referirían al instituto en sus primeros años esencialmente como un "punto de encuentro", una institución que, independiente de los contenidos allí impartidos, servía a una nueva generación de cinéfilos como un lugar en el que dar con otras personas que compartían una misma pasión. Basilio Martín Patino, hablaba en estos términos:

Había muy buenos amigos, charlábamos, había un ambiente estupendo, pero nunca porque hubiera buenos profesores, porque hubiera sistemas académicos, medios de producción, prácticas, nada de eso. Lo único éramos nosotros, que nos reuníamos, que teníamos que dar un sentido crítico, que teníamos una entrañable amistad que no se romperá nunca (Blanco Mallada, 1990: 166-167, anexos).

Juan Antonio Bardem, estudiante de la primera generación, comentaba así la impresión que le causó comprobar que no estaba solo, que formaba parte de un grupo generacional más amplio fascinado por el cine: "me quedé asustado, porque yo estaba completamente convencido de que el único que se interesaba en el cine en toda España era yo y me encontré allí con cientos de personas..." (1964: 27).

Este topos narrativo se mantendrá a lo largo de los años, independientemente de las distintas etapas, directores o generaciones que visiten sus 
aulas: el IIEC, como desde 1962 la Escuela Oficial de Cinematografía, será ante todo un punto de encuentro entre iguales, fascinados por el cine, en el que se tiene acceso a unas obras que no se encuentran en las salas comerciales. Los estudiantes de 1950 forman una generación que en 1959 José María Pérez Lozano se atrevió a definir:

(...) asombra la coincidencia. Porque la mayoría de edad, los 21 años, nos llegan -1948- en un momento decisivo, sobre todo para el cine italiano. Ya vamos al cine con novia formal, dejando los libros de las oposiciones abandonados. Unos chicos fanfarrones entran en el IIEC -Bardem, Berlanga, Soria, Maesso...- pero nosotros, aún distantes de ese mundo -alguno, uno mismo, iniciándose en él tímidamente- empezamos a soñar con el cine (Pérez Lozano, 1959: 3).

La cinefilia en este ambiente de carestía lleva a la utilización del instituto desde un primer momento como centro en el que, independientemente de la labor docente, se potencian distintas actividades culturales. Ciclos de cine amateur -ya en 1948-, conferencias o la publicación de textos de los nuevos estudiantes en revistas como La Hora. Semanario de los Estudiantes Españoles - publicado por la Jefatura Nacional del SEU-, Índice, Ínsula u Objetivo empiezan a tejer una red que será esencial para la aparición de una importante subcultura cinéfila a partir de finales de los años cuarenta. Mientras que la cinefilia que habría de desarrollarse en Francia durante esos mismos años lo hará enfrentada a la institucionalización del cine representada por el IDHEC, homólogo francés del IIEC. En el caso español el centro será uno de los nudos esenciales dentro de una red que empieza a extenderse esos primeros años: fundación de la Filmoteca Nacional, formación de los directores del Nuevo Cine Español, desarrollo de una doctrina estética en torno a Nuestro Cine a partir de 1961...

En esos momentos, la pasión cinéfila de las nuevas generaciones cristaliza sobre todo en el visionado de películas situadas más allá del mainstream. El interés por aquellas obras alejadas de los títulos que se pueden ver en las pan- tallas comerciales se alimenta ya desde los primeros años con proyecciones de los films que profesores como Victoriano López, Luis Gómez Mesa o Carlos Fernández Cuenca habían podido ir reuniendo a lo largo de sus carreras, eventos que ponen la base de la futura labor del cineclub. Este es también el descubrimiento de un cine leído: será en el IIEC donde a lo largo de los años cincuenta los estudiantes tendrán acceso a publicaciones, libros y revistas especializadas que los conectan con los debates internacionales. En 1951 hay ya un arranque de biblioteca que empieza a acumular guiones, indispensables para los alumnos y que en menos de dos años llegará a alcanzar los 300 ejemplares 7 . También libros y revistas nacionales y, sobre todo, extranjeros, que plantean una nueva manera de ver y analizar el cine apuntando su labor de crítica social, redescubriendo a autores, ensayando nuevas formas de mirar a la realidad; son las cabeceras de referencia que empiezan a aparecer en estos años como Cahiers du cinéma (1951), Positif (1952), Cinema Nuovo (1952), Film Culture (1954) y desde la misma fundación del centro, la Revue Internationale de Filmologie . $^{8}$

Este "cine en papel" (Nieto Ferrando, 2009) remite en muchas ocasiones a obras conocidas solo en parte, que actúan como referente in $a b$ sentia de un discurso en el que muchas ocasiones las películas son leídas sin haber podido ser vistas. Son así especialmente significativas las crónicas aparecidas en varios números del boletín escritas por el estudiante Enrique Pinilla. Este, aprovechando una estancia en París en las

\footnotetext{
7 Ver la referencia en el número seis del boletín y el NO-DO Instituto de Investigaciones y Experiencias Cinematográficas de 1 de enero de 1953.

8 Interesante es en este sentido una lista con los libros que la Dirección General de Cinematografía y Teatro se plantea adquirir en 1956 para la biblioteca del instituto. Más allá de los títulos nombrados son especialmente significativos los comentarios al margen acusando/tachando publicaciones por ser "propaganda comunista". Curiosamente el problema no lo suelen plantear los clásicos soviéticos - las obras de Dziga Vertov o Sergei Eisenstein se pueden considerar a estas alturas ya canonizadas- sino los autores contemporáneos italianos: Il cinema e l'uomo moderno de Umberto Barbaro o Cinema quinto potere de Luigi Chiarini (AGA Caja 19761, 19768).
} 
Navidades de 1950, visita con fruición la Cinémathèque y diversos cineclubs en los que visiona los clásicos de la historia del cine. El boletín incluirá durante los siguientes meses descripciones de obras como El arsenal (1928) y Tierra (1930) de Aleksandr Dovzhenko, iQué viva México! de Sergei Eisenstein (1931), Mark Semionovich Donskoi (Et l'acier fut trempé, 1942), D. W. Griffith (Intolerancia, 1916, que ve en la Cinemateca), La pasión de Juana de Arco (1928) y Días de Ira de Carl Theodor Dreyer (1943), algunos "films de arte" de Luciano Emmer, obras de Alain Resnais así como Works of Calder (1950) de Herbert Matter.

\section{PRÁCTICAS Y DEBATES DEL INSTITUTO: EL CINECLUB Y LA FILMOLOGÍA}

En este ambiente de fascinación y escasez aparece el cineclub. Para darle un nombre, los alumnos se fijan en un pionero del cine nacional de principios del siglo XX, Segundo de Chomón, pero la mirada hacia los primeros años de la historia del cine no se queda aquí. Desde el primer momento sus responsables, pese a las limitaciones impuestas por la carestía, buscan un programa que ofrezca nuevas perspectivas de diferentes etapas históricas de acuerdo con la clase en la que esta actividad se encuadra: Historia del Cine, impartida por Carlos Fernández Cuenca que, además en esos años, acaba de publicar el sexto volumen de su Historia del Cine, iniciada en 1948.

La iniciativa cineclubista dentro del IIEC se presenta como un proyecto coral y empieza con entusiasmo. Ha de crear el ambiente para "ordenadas discusiones al final de las proyecciones, examinándose técnicas y estilos, etapas históricas, tendencias y problemas fundamentales en orden a la creación artística”, de acuerdo con las palabras de Victoriano López en el segundo número del boletín, publicación que, a la larga, habría de proporcionar la base de una revista interna de más empaque para todo el instituto, lugar de reflexión teórica y órgano de discusión. El boletín comienza, no obstante, dubitativo, con un primer número de diez páginas, que aumentarán a lo largo del segundo semestre del curso académico hasta asentarse en unas veintiuna o veintidós páginas. Se distribuye durante las sesiones del cineclub y su producción es todavía artesanal. Faltan medios: se escribe en un papel de baja calidad, con una máquina que carece de las grafías auxiliares del castellano. La encuadernación, con grapas, revela lo rudimentario de la empresa, así como también la ausencia de fotografías, elementos tipográficos, etc. No obstante, el proyecto es ambicioso y va más allá de las actividades exclusivas del cineclub, erigiéndose en sus pocos meses de vida en la publicación de referencia del IIEC. Y es que si la cinefilia clásica no se reduce a ver películas e incluye además un aspecto discursivo esencial -ese "hablar sobre el cine y extender este discurso" que menciona Antoine de Baecque (2003: 11)-, el cineclub va de la mano del boletín como proyecto cinéfilo: un proyecto sui generis que incluye entrevistas, textos teóricos, traducciones de autores extranjeros, reflexiones sobre los problemas en el día a día de la escuela, las críticas de las películas vistas y también de aquellas que pudieron ser simplemente leídas, protestas de los alumnos o los planes de crear una cineteca en base al archivo del instituto y que solo un par de años más tarde darían lugar a la aparición de la Filmoteca Nacional.

Por sus páginas pasan profesores como Victoriano López, Fernández Cuenca o Mauricio de Begoña. Se discuten los problemas más acuciantes de los alumnos y se presentan textos que serán fundamentales ya que nos encontramos en un ambiente de carestía general en el que la base para la mayoría de las clases la constituyen los apuntes de los propios alumnos ${ }^{9}$. También se publican otros ensayos como un extracto de $L a$ Televisión y el Cine de René Clair, aparecido originalmente en enero de 1951 en Television and Cinema (número 3); reflexiones cortas, de carácter aforístico, provenientes a menudo de la ya extinta Cine Experimental; o fragmentos de Film

9 Entrevista a Eduardo Ducay del 17 de diciembre de 2015. 
Technique de Pudovkin traducidos por Eduardo Ducay.

La responsabilidad de la realización del boletín recae en un grupo relativamente pequeño de autores. Estos intentarán de forma continuada aumentar el grupo de colaboradores con abundantes llamadas para que otros estudiantes manden sus textos. Parece que carecen de éxito pues la mayoría de los artículos vienen firmados por Rafael Tapia Arroyo, Enrique Pinilla ${ }^{10}$ y, sobre todo, Eduardo Ducay. La figura de Ducay es especialmente significativa. Llega a Madrid en 1950 para estudiar en el IIEC, y unos meses después se pone en marcha el cineclub. Este es para él la continuación natural de las actividades iniciadas cinco años antes en su Zaragoza natal. En diciembre de 1945, tomando como modelo el Cineclub del Círculo de Escritores Cinematográficos y al amparo de la falangista Delegación de Educación Popular, ya había fundado el Cineclub Zaragoza, pionero y referente durante al menos las dos décadas entre los círculos cinéfilos españoles (Hernández Marcos y Ruiz Butrón, 1978: 39f).

Además, en el periodo en el que arranca el cineclub, Ducay empieza a colaborar en la página de cine de la revista cultural Ínsula con un texto que dedica a René Clair en abril de 1951, "René Clair, en perspectiva”. Unos meses más tarde, extiende su colaboración a Índice, revista cultural de carácter disidente, en la que se ocupará de la sección de cine al alimón con un Ricardo Muñoz Suay que está en estos años intentando reactivar la actividad cultural del Partido Comunista en España. Este grupo será el que en 1953 ponga en marcha Objetivo y en 1955 lance las Conversaciones de Salamanca ${ }^{11}$. Son a través de estos contactos con el campo cultural-literario desde donde empiezan a formularse alternativas estéticas que habrían de tener su reflejo en algunos de los trabajos apoyados en un Realismo Crítico que nacen en el IIEC. Si bien la presencia política del PCE tendría continuación en el propio centro en torno a un grupo que incluirá a Julio Diamante y, en los años siguientes, a gente como Basilio Martín Patino o Fernando Sánchez Dragó. Sin embar- go, el profesor Antonio del Amo, que había sido miembro del Partido Comunista y publicado en Nuestro Cinema durante los años treinta, rehúye el contacto con los estudiantes al tratar estas cuestiones $^{12}$.

La base del programa del Segundo de Chomón, de forma similar a otros cineclubs -en esta época todavía escasos en España-13, la forma obras de organismos culturales como embajadas, legaciones, institutos culturales, etc. Con ello se busca un programa alternativo a aquel que se puede encontrar en las salas comerciales, con especial interés en un cine de vanguardia y con una perspectiva claramente histórica. Hay también motivos económicos, pues la mayoría de las películas ofrecidas por las instituciones anteriormente señaladas las consiguen gratis. La primera sesión tiene lugar el uno de febrero de 1951: tras una introducción a cargo de Fernández Cuenca se proyecta la obra de vanguardia Sinfonía en color (Ein Spiel in Farben, 1934) de Oskar Fischinger, el corto documental Pacific 231 de Jean Mitry, que acababa de ganar la Palma de Oro en Cannes en 1949), Le six juin a L'aube de Jean Gremillon (1945), Sinfonía en Azul (Komposition in Blau, 1936), también de Fischinger, así como el documental etnográfico Hierba (Grass) de Me-

\footnotetext{
10 Se trata del compositor y director de orquesta Enrique Pinilla Sánchez-Concha; nacido en Perú, pero con doble nacionalidad peruana-española, estudió en el instituto. En 1958 consigue diplomarse en composición en el Conservatorio de Madrid. A lo largo de su carrera escribió música para el cine.

11 En el apartado octavo de las conclusiones de las Conversaciones -Formación Profesional-, se hace referencia explícita a los problemas del IIEC y se plantean cinco medidas centradas sobre todo en la integración del centro en la industria. Ver Objetivo, 1955, núm. 6, p. 36.

12 Entrevista con Eduardo Ducay celebrada el 17 de diciembre de 2015.

13 En abril de 1952 tiene lugar en Madrid un primer Congreso Nacional de Cineclubs en el que participan veintiséis de estas organizaciones. En este marco se plantean nueve ponencias; los cineclubs reunidos plantearon sobre todo la necesidad de que sus actividades, dirigidas a un público más específico, estuviesen protegidas de las formas más rigurosas de censura (Martínez-Bretón, 1987: 130). A esta iniciativa organizativa le habría de faltar continuidad en los siguientes años y tendría que ser reactivada en 1957 en forma de la Federación Nacional de Cineclubs. Véase en este sentido el texto de Hernández Marcos y Ruiz Butrón (1978.: 43).
} 
rian S. Cooper y Ernest Schoedsack (1925). El catorce de febrero, esta vez tras una presentación del profesor de Caracterología Antonio del Amo, se muestran Van Gogh de Diehl y Resnais (1948), H. Rousseau de Lo Duca (1950) y María de Paul Fejos (1932). En este caso es Antonio Ceballos el encargado de moderar los comentarios que siguen a la proyección.

El cinco de marzo, de nuevo con una presentación de Fernández Cuenca, se ve el documental de la Scottish Office of Information Children of the City (1944) de Budge Cooper y producido por Paul Rotha, aunque el plato de la fuerte no solo de esta sesión sino también de estas primeras proyecciones es Tempestad sobre Asia de Pudovkin (1928) ${ }^{14}$. Esta pasa así a ser la sesión más concurrida, en la que se pueden apreciar "la presencia de algunas personas de las que nadie podía dar razón. Atraídas, sin duda, por el aroma de lo prohibido" como comenta Ducay en el cuarto número del boletín. Y continúa: "Presentó el programa nuestro profesor de Historia del Cine -se refiere a Fernández Cuenca-, quien hizo constar antes que nada el carácter exclusivamente pedagógico que rodeaba a la presentación del famoso film de Pudovkin”. No obstante parece que Ducay no se puede reprimir cuando, cerrando el párrafo que describe la presentación, comenta: "Por otro lado, hay en la película un aspecto político interesante como postura, característico de todo el cine ruso de la gran época".

Si bien el cineclub forma parte de las actividades ofertadas en torno a las clases de Historia del Cine de Fernández Cuenca, desde el inicio vemos como pasa a instituirse como una zona de negociación de poder e influencia entre la dirección del instituto y los alumnos, que buscan llevar la iniciativa en la preparación de las proyecciones y la discusión de las obras. Ducay también plantea la oportunidad de que sean los estudiantes los que lleven las discusiones y no Fernández Cuenca, pues "no cabe duda de que, si la cosa es hecha por nosotros, las discusiones serían mucho más animadas" ("III Sesión del cineclub Segundo de Chomón" en el cuarto número del boletín).
La cuarta sesión del cineclub, que tiene lugar el 28 de marzo, está dedicada a las películas documentales proporcionadas por la Embajada de los EEUU. Son cortometrajes de tipo educativo e industrial: Cooperativas eléctricas, Las materias plásticas, Ha nacido un barco así como El enemigo invisible y La semilla de oro, estas dos últimas producidas por Walt Disney. Con estos títulos los estudiantes pierden el interés. La asistencia es menor, como lamenta Rafael de Tapia Arroyo en el quinto número del boletín. La siguiente sesión tiene lugar el miércoles 11 de abril a las 5 de la tarde y la presentación corre de nuevo a cargo de Fernández Cuenca. Se muestran Fecundidad -de Alberto Carles Blat, del IIEC- así como la película húngara Primavera Mortal, basada en la novela Lajos Zilhay, y cuya copia ha sido prestada por la Legación de Hungría. La sexta sesión tiene lugar el 25 de abril a las cinco de la tarde. La introduce Joaquín Granados, que muestra primeramente dos películas en $16 \mathrm{~mm}$ propias y también dirigirá la discusión final. Se proyecta el clásico del expresionismo alemán Sombras (Schatten, 1923) de Robinson y Lot in Sodom, el cortometraje experimental producido por James S. Watson en 1933.

Estos títulos nos proporcionan una muestra bastante significativa de la dieta cinematográfica a la que tienen acceso los estudiantes del instituto. $\mathrm{Al}$ retomar la línea argumental anteriormente expuesta referida al aspecto discursivo de la cinefilia, cabría plantear si podemos hallar en estos primeros años, y en paralelo a las prácticas de exhibición, una línea teórica más o menos sistemática en torno al IIEC. No lo parece a primera vista: el centro concibe su formación desde un principio con un carácter preeminentemente práctico, supeditada al objetivo de encontrar para sus estudiantes un trabajo dentro de la industria; en la tenue reflexión en Cine Experimental o en la obra ensayística de profesores como Fer-

14 Se trata con toda seguridad de la copia que, a partir de la fundación de la Filmoteca en 1953 circularía con gran asiduidad entre los cineclubs y que, de acuerdo con Román Gubern (Gubern, 1997: 101), Fernández Cuenca presentaba a cambio de los gastos de viaje y un honorario por la conferencia. 
nández Cuenca apenas encontramos meditaciones capaces de sustentar un discurso teórico, una situación que en lo esencial casi no variaría a lo largo de los siguientes veinticinco años, si bien la aparición de la revista Nuestro Cine en 1961 se puede entender, por las más que evidentes relaciones personales, como foro teórico-crítico del IIEC.

Sorprende por su excepcionalidad la presencia de la Filmología en el Instituto en la década que va de 1948 a 1957, situada en los planes de estudio en las clases de segundo año ${ }^{15}$. En ella encontramos un intento de dotar al instituto de una base teórica basándose en una disciplina contemporánea con importante presencia internacional. Es una de las pocas vetas del debate sobre teoría del cine que llega a nuestro país sin retrasos, alcanzando además cierta importancia institucional, no solo en el IIEC sino también en el Consejo Superior de Investigaciones Científicas y en la nueva Asociación Española de Filmología ${ }^{16}$.

El camino por el que llega la Filmología al instituto es por otro lado bastante prosaico. José Gutiérrez Maesso, alumno de la primera promoción, apunta que esta importación de la Filmología se debe a un primer viaje, iniciático, que él realiza en el verano de 1947 a París y en el que toma contacto con revistas y libros de cine. Con el viaje quería paliar su falta de conocimientos cinematográficos tras haberse comparado con otros estudiantes matriculados en la escuela. Cuando vuelve de la capital francesa, consciente de la novedad que plantea la Filmología, se dirige a Serrano de Osma para plantearle la necesidad de incorporar esta nueva corriente dentro del instituto (García de Dueñas, 2003: 106f). De hecho, será Maesso, en una curiosa combinación que nos dice mucho de la singular organización del centro y la improvisación de los primeros años, el encargado de propagarla en un doble papel de alumno y profesor al mismo tiempo. La figura esencial en este contexto de importación y adaptación de la Filmología sería no obstante el fraile capuchino Mauricio de Begoña, polígrafo, censor y profesor en el instituto tras la renuncia de Maesso, que adapta los principios expuestos por Gilbert Cohen-Séat en sus Essais sur les principes d'une Philosophie de cinéma en 1946 y en un primer congreso "filmológico" celebrado en el París de postguerra. En 1948 la asignatura Introducción a la Filmología pasa a formar parte de los planes de estudios de los alumnos de segundo año y se crea la cátedra de Filmología, que asume de Begoña (Nieto Ferrando, 2012: 860f).

Los aspectos más espirituales de la Filmología -una "disciplina" de fondo humanístico pero con aspiraciones holísticas que abarcan la filosofía, psicología y sociología del cine -17 facilitan su asimilación en el contexto nacional-católico $\mathrm{y}$ concretamente en un instituto que en estos primeros años es todavía excesivamente dependiente de los aspectos técnicos de la enseñanza y en el que falta un poso de reflexión teórica. Encontramos aquí similitudes con el caso francés donde en estos años la Filmología sería la corriente principal sobre la que se habría de sustentar la progresiva sistematización y posterior institucionalización de los estudios de teoría ci-

15 El plan de estudios introducido en el curso 1957-58 llegó a la plena implantación en el curso 1960-61, periodo durante el cual los alumnos antiguos continúan estudiando de acuerdo con el plan de 1947. Véase: (Blanco Mallada, 1990: 85f).

16 Ya en 1949 se funda la Asociación Española de Filmología, cuyo director será José Germain, en la que el instituto sigue estando fuertemente representado: Carlos Fernández Cuenca es nombrado vicepresidente; Carlos Serrano de Osma y Mauricio de Begoña actúan como vocales junto a gente como Julián Marías o Fernando Fernán Gómez. Un año después, en 1950, se celebra en Madrid la I Semana de Filmología, en la que se participa el propio Cohen-Séat. En 1952 aparece el primer número de la Revista Internacional de Cine, que continúa la línea de una crítica cinematográfica católica, $y$, al mismo tiempo - lo cual no es ni mucho menos normal en este periodo-, orientada a los movimientos internacionales. También en 1952 se funda en el CSIC un departamento de Filmología en el que de nuevo encontramos, como asesores, a Mauricio de Begoña y Feliciano López. De Begoña publica en 1953 con el apoyo de la Dirección General de Cinematografía y Teatro sus Elementos de Filmología. Teoría del cine, que habría de servir de libro de texto en el instituto. No obstante, tanta actividad no habría de bastar para afianzar la asignatura, que desaparece en 1957 con la reforma del plan de estudios.

17 Véase en este sentido el estudio ya canónico de Edward Lowry The Filmology Movement and Film Study in France (1985) así como, en fechas más recientes, el texto de Martin Lefebvre L'aventure filmologique: documents et jalons d'une histoire institutionelle (2009: 59-100). 
nematográfica. Sin embargo, los alumnos consideran esta asignatura en general una pérdida de tiempo: "Creemos que la Filmología constituye un cuerpo de conocimientos de orden filosófico, y que verdaderamente a los alumnos de especialidades puramente técnicas solo les servirá, en el mayor de los casos, de quebradero de cabeza" comenta Eduardo Ducay (El Instituto y nosotros, en el segundo número del boletín).

Es interesante considerar esta crítica a la Filmología en un contexto más amplio pues plantea interesantes reflexiones sobre la evolución de la disciplina y su relevancia durante la siguiente década. Por ejemplo, André Bazin publica solo unos meses después del texto de Ducay, bajo el seudónimo Florent Kirsch en el quinto número de los Cahiers du cinéma un artículo sobre la Filmología en el que ataca a Cohen-Séat - "que hace de su desinterés [por el cine] una virtud intelectual" - y en el que también recomienda con cierto sarcasmo a los filmólogos no ir al cine "pues quizás lo que allí aprendan solo cause confusión en su ciencia" (Kirsch, 1951: 36). Y es que pese a su especificidad, estos dos ejemplos nos remiten a un contexto mucho más amplio: la Filmología conquista en estos años el IIEC, el CSIC y la española Revista Internacional del cine (1952-1963) y al mismo tiempo en Francia se hace fuerte en el nuevo Institut de Filmologie de la Sorbona y en la Revue internationale de filmologie (1947-1961). En ese momento, las nuevas generaciones de críticos y cineastas -la nouvelle critique francesa en torno a los Cahiers de Bazin o los propios estudiantes del IIEC-, interesadas en un enfoque más apasionado, filmico, cinéfilo del cine y sus obras, rechazarán la respetabilidad académica de la Filmología y su aparente desinterés por la historia y la estética cinematográfica. Habrán de ser pues otros enfoques, más cercanos un esteticismo formal o un realismo crítico, las encargados de acompañar a estas nuevas generaciones que propiciarán el nacimiento de los Nuevos Cines. La Filmología perderá no solo representación en las instituciones anteriormente señaladas: además, ninguna de las publicaciones especializadas que desde mediados de los años cincuenta plantean una renovación en el campo de la crítica cinematográfica la asimilará como paradigma crítico. A principios de los años sesenta el proyecto filmológico entendido como línea crítica de cierta relevancia está ya casi olvidado.

Por ello es significativa la posición de la Filmología en IIEC en 1951: cuando Mauricio de Begoña es entrevistado en el sexto número del boletín del cineclub sus reflexiones, abstractas y grandilocuentes, a menudo parecen vacías: "La filmología no es la sustancia del cine, sino el concepto de esta sustancia. Su actitud es contemplativa, y no tiene más eficacia en el cine que la grandísima que toda contemplación ejerce sobre la práctica". Ello no hace más que probar una desconexión con los intereses de sus alumnos. De hecho, y como también se verá en la publicación de sus Elementos de Filmología en 1953, en la que en un libro de cuatrocientas apenas hay referencias a películas, no hay interés por tratar aspectos específicos de la producción o análisis cinematográfico, parece que la Filmología está más interesada en la especulación teórica que en su aplicación empírica. En la propia entrevista en el boletín, el movimiento más en boga en aquellos momentos, el Neorrealismo italiano, es despachado por Begoña con argumentos que denotan una falta de interés concreto por el cine más actual: "Este neorrealismo lo considero transitorio. Más bien como una moda que no tardará en pasar. (...) [¿]Por qué se entiende por realismo la exposición acre de la vida? Hay otras infinitas cosas menos acres, que no se incluyen en él”.

La referencia al Neorrealismo no es casual. Se trata del modelo del nuevo cine, omnipresente en publicaciones culturales y en las páginas de cine de semanarios. También omnipresente de forma indirecta, hay que apuntar, pues las obras esenciales de este nuevo movimiento apenas pueden ser vistas en España en estas fechas. Será a partir de Primera Semana del Cine Italiano, celebrada por el Instituto de Cultura Italiana de Madrid solo unos meses después de la aparición del cineclub, cuando los grupos cinéfilos de la capital empiecen a descubrir las obras más re- 
levantes de este movimiento. Mientras tanto, la recepción -esencialmente discursiva- del Neorrealismo se adapta a las realidades culturales del franquismo, sin tener en cuenta por ejemplo los por entonces en España desconocidos títulos de Roberto Rossellini al tiempo que toma como referencia obras de un neorrealismo cada vez más "rosa" producidas desde principios de los cincuenta y pergeñando una espiritual versión de base católica que anticipa el neoidealismo de los primeros años, hasta aproximadamente 1961, de la revista católica Film Ideal (Nieto Ferrando, 2012: 865f).

Las referencias más o menos directas a la nueva corriente las encontramos en el boletín. También en un texto corto de José María García de la Rasilla haciendo referencia al Neorrealismo titulado "Misión y Significado de una nueva Tendencia”. Junto a Milagro en Milán de Vittorio de Sica, el texto menciona positivamente otras obras de un cine católico que difícilmente pueden ser catalogadas como neorrealistas: Primera comunión / Una hora en su vida de Alessandro Blasetti (1950), o Cielo sobre el pantano de Augusto Genina (1949). Interesante, por significativo de la forma en la que habrá de producirse una parte importante de la recepción neorrealista en la próxima década, es el acento que pone en "en un realismo más humano y espiritual, es decir, poetizado" (número tres del boletín). Al tiempo que se aleja de los aspectos documentales del movimiento:

Vemos pues, como su grosero y crudo trazado de la vida se ha elevado, al conjuro de una visión poética de la realidad. [...] Ahora es cuando podemos sentir el Arte de esta nueva tendencia llamada "neorrealista", en su actual concepción, pues aun basándose en hechos de la vida, los transforma - sin embargo- en formas de belleza con la intención de emocionarnos estéticamente, y yo creo que también con la de despertar el amor al que se llegará -al menos en gran parte- por el lirismo que se ha incrustado en esta nueva tendencia, para que estos hechos humanos no constituyan un fin por sí mismo (Nieto Ferrando, 2012: 865f).

\section{CONCLUSIONES}

Si bien a los pocos meses de iniciado el proyecto de cineclub Segundo de Chomón desaparece víctima de las dificultades materiales y de la falta de interés del alumnado ${ }^{18}$, actividades similares -sobre todo las famosas proyecciones de los sábados durante los años sesenta- jalonarían la vida del IIEC y de la EOC hasta su desaparición. De un modo que conecta con el papel que habrían de jugar qué instituciones análogas en países en los que el acceso a películas estaba restringido por condicionantes tanto económicos como políticos - por ejemplo la FAMU, fundada en Praga un año antes que el centro español-, el instituto y la escuela se convertirían, con independencia de una enseñanza de más o menos calidad, en centros cinéfilos por excelencia: instituciones estatales en las que era posible ver un cine inaccesible para la gran mayoría de los espectadores, a la vez que se mantenía un contacto con una comunidad de iguales. Instituciones también en las que en su excepcionalidad daban lugar a espacios de cierta negociación de poder e influencia. El ejemplo del cineclub nos lo muestra a pequeña escala, como iniciativa que parte de los estudiantes y que buscará liberarse de la influencia de los profesores en las presentaciones y discusiones; a la larga y aun sin salirnos del periodo aquí analizado, esta iniciativa dará lugar a los conflictos en el periodo 1954-55 provocados por la falta de instalaciones adecuadas y que precipitarán la destitución de Victoriano López.

El boletín publicado de forma paralela a la celebración de las actividades del cineclub ilustra las iniciativas de este a la vez que aspira también a presentarse como órgano de reflexión crítica y teórica del instituto, con poco éxito. No obstante, su labor es significativa como reflejo de los intereses de los alumnos. Con la referencia puntual a la Filmología y la forma

18 Y es que "había muy poca gente porque, por raro que parezca, a los alumnos el cine antiguo no les interesaba" (Entrevista con Eduardo Ducay, 17 de diciembre de 2015). 
en la que su presencia en el centro fue recogida en las páginas del boletín este artículo ha querido además apuntar algunas características $-\mathrm{y}$ aporías- del proyecto filmológico dentro del ambiente cinéfilo de la escuela.

El acercamiento propuesto en estas páginas, parcial por la escasez documental, ha de entenderse dentro de sus limitaciones como una invitación a repensar la labor del instituto y de la escuela desde una perspectiva más cercana a una historia cultural del cine. Un enfoque que, al tiempo que permite encuadrar esta historia dentro de una perspectiva transnacional, nos sirve para comprender su relevancia, más allá de su labor formativa, como uno de los pilares básicos de la cultura cinematográfica española hasta principios de los años setenta. 


\section{BIBLIOGRAFÍA}

DE BAECQUE, Antoine. La Cinéphilie. Invention d'un regard, histoire d'une culture 1944-1968, Fayard, París, 2003.

BARDEM, Juan Antonio. "Bardem, 64. Confesiones a las cinco de la tarde", Nuestro Cine, núm. 29 (mayo 1964), pp. 24-40.

BLANCO MALLADA, Lucio. IIEC y EOC: una escuela para el cine español, [Tesis Doctoral], Universidad Complutense, Madrid, 1990.

FILMOTECA ESPAÑOLA (Ed.). 50 años de la escuela de cine. Cuadernos de la Filmoteca, núm. 4, Filmoteca Española, Madrid, 1999.

GARCÍA DE DUEÑAS, Jesús. José G. Maesso, el número 1, Diputación de Badajoz, Badajoz, 2003.

GARCÍA ESCUDERO, José María. “El Director General de Cine cuenta la historia del IIEC", Film Ideal, núm. 109 (diciembre 1962), pp. 676-679.

GUBERN, Román. Viaje de Ida, Anagrama, Barcelona 1997.

HEREDERO, Carlos F. Las huellas del tiempo: cine español, 1951-1961. Generalitat Valenciana, Valencia, 1993.

HERNÁNDEZ MARCOS, José Luis y RUÍZ BUTRÓN, Eduardo A. Historia de los Cine Clubs en España, Ministerio de Cultura, Madrid, 1978.

KIRSCH, Florent [André Bazin]. "Introduction à une filmologie de filmologie", Cahiers du cinéma, núm. 5 (septiembre 1951), pp. 33-39.

LEFEBVRE, Martin. "L'aventure filmologique: documents et jalons d'une histoire institutionelle", Cinémas, núm. 19, 2-3 (2009), pp. 59-100.

LOWRY, Edward. The Filmology Movement and Film Study in France, Michigan University Press, Ann Arbor, 1985.

MARTÍNEZ-BRETÓN, Juan Antonio. Influencia de la Iglesia Católica en la cinematografía española (1951-1962), Harofarma S.A., Madrid, 1987.

MONTERDE, José E. y PIÑOL, Marta (Ed.). Crónica de un desencuentro. La recepción del cine moderno en España. Ediciones de la Filmoteca, Valencia, 2015.

NIETO FERRANDO, Jorge. Cine en papel. Cultura y crítica cinematográfica en España [1939-1962], Ediciones de la Filmoteca, Valencia, 2009.

NIETO FERRANDO, Jorge. "La reflexión y la crítica católica en la prensa cinematográfica bajo el franquismo. Del nacional-catolicismo a Ingmar Bergman", Estudios sobre el Mensaje Periodístico, Vol. 18, núm. 2 (2012), pp. 855873.

PÉREZ LOZANO, José María. "Una generación frente al cine", Film Ideal, núm. 31 (1959), pp. 3 y 26.
RODRÍGUEZ TEJADA, Sergio. "The anti-Franco student movement's contribution to the return of democracy in Spain", Espacio, Tiempo y Educación, Vol. 2, núm. 2, juliodiciembre 2015, pp. 77-106.

SALVADOR MARAÑÓN, Alicia. De iBienvenido Mr. Marshall! a Viridiana. Historia de UNINCI: Una productora española bajo el franquismo, Egeda, 2006. 